\title{
Multi-Agent Rendezvous Problem Based on State Backtracking
}

\author{
Jiahe $\operatorname{Tian}^{\text {a) }}$ \\ Computer College of Guangdong University of Technology, Guangzhou,510000, China. \\ a) Corresponding author: 576152161@qq.com
}

\begin{abstract}
This paper studies a new distributed multi-agent rendezvous problem based on state backtracking. It uses the historical state information of the agent as a reference for the current time to achieve the purpose of preserving topology connectivity and accelerating the rendezvous speed. The agent uses different switching strategies according to the changes of the historical moments and surrounding neighborhoods at the current moment and uses the potential function to enhance the topology connectivity so that the agents eventually rendezvous at the same point. Finally, the simulation experiment and the rendezvous effect statistics are given. The simulation experiments with 50 agents randomly distributed in the plane are taken as examples and the effects are compared. The results show that under this protocol, multi-agent systems can achieve gradual rendezvous at the same point.
\end{abstract}

Key words: multi-agent; rendezvous; distribute system; cooperative control; state backtracking.

\section{INTRODUCTION}

In recent years, multi-agent system consistency has become a hot topic in multi-agent distributed coordination control and has received extensive attention from researchers in various fields. Multi-agent system consistency means that in the limited time, a number of individuals operating autonomously in the network environment through the local coordination and cooperation, so that the status of the agent eventually reached a consistency. As a typical application of multi-agent consistency problem, multi-agent rendezvous problem has broad application prospects in fields such as military, aerospace, unmanned aerial vehicles, and industrial production.

In the 1990s, Ando et al. [1] first raised the issue of multi-robot rendezvous problem attracted much attention. Lin et al [2]. conducted in-depth research on synchronous and asynchronous multi-agent rendezvous problems, Dimos V. Dimarogonas and Karl H. Johansson [3] designed a discontinuous distributed feedback control protocol for nonholonomic ally constrained agents, allowing the agent to reach a clustering point at the same time while maintaining topology connectivity. Qing Hui [4] developed a new coordination framework for finite-time aggregation in dynamic networks based on system thermal dynamics and finite-time semi-stability theory.

The above research results have achieved multi-agent progressive aggregation control, however, the movement state of the next moment in the agent depends on the current moment itself and the neighbor's movement state and without reference to the historical state of the previous moment, based on the researches of Housheng Su [5] and Michael M. Zavlanos et al. [6], this paper proposes a new state backtracking aggregation control algorithm that maintains topological connectivity. Each agent keeps the historical information of last time about itself and neighborhood and used as a reference for the motion conditions of the next moment. 


\section{THEORETICAL BASIS AND PROBLEM DESCRIPTION}

In a system of $N$ agents that can move autonomously in the plane, each agent can continuously track other agents' motion states within their own sense distance and based on the sensed motion of these agents autonomously planning their movement, so that the agent achieves the established goal [7].

In the multi-agent rendezvous model, it is assumed that the agents have the same sensing range $r$ and the network topology is $G=(V, E)$, where $V=\left(v_{1}, v_{2}, \cdots, v_{n}\right)$ is the set of nodes (i.e., agents in the graph), and $E \in V^{2}$ represents the set of edges consisting of node pairs.

If there is information transfer between the agent $i$ and $j$, there is a connection between agent $i$ and $j$, and $\left\|x_{i j}\right\|=\left\|x_{i}-x_{j}\right\|$ represents the distance between the agent $i$ and agent $j$. Generally use $A=\left[a_{i j}\right]$ to represent adjacency matrix of graph $G$, where $a_{i j}=1$ that $(i, j) \in E \wedge\left\|x_{i j}\right\|<r$,otherwise $a_{i j}=0$.

In the discrete time, the multi-agent distributed rendezvous protocol is as follow for agent $i$ :

$$
x_{i}(t+1)=x_{i}(t)+\varepsilon \sum_{j \in N_{i}} a_{i j}\left(x_{j}(t)-x_{i}(t)\right), t \geq 0
$$

Where $\varepsilon$ is the adjustment factor of the agent used to adjust the adjustment factor for each movement, and $N_{i}$ used to represent the set of neighbor points within the communication range $r$ of the agent $i$, i.e. $N_{i}=\left\{j \mid\left\|x_{i j}\right\|<r\right\}$.

Definition 1: When all agents satisfy the condition $\left\|x_{j}(t)-x_{i}(t)\right\| \rightarrow 0, \forall i, j \in V \wedge t \rightarrow \infty$, it is regarded as multi-agent system (3) position state rendezvous [2].

Through the information of multi-agent historical moments, it is possible to predict the trend of the movement of the agents so as to adopt different measures to achieve the purpose of maintaining topology connectivity.

Definition 2: The agent $i$ calculates the distance $\left\|x_{i j}(t)\right\|$ from the current moment of the neighbor agent $j$ and the distance $\left\|x_{i j}(t-1)\right\|$ from the previous moment. If the distance between agent $i$ and agent $j$ is increased, i.e. $\left\|x_{i j}(t)\right\|-\| x_{i j}(t-1)||>0$, the agent $j$ is considered to have a tendency to break away, and a trend of rendezvous otherwise.

Control Method 1: As shown in the classical multi-agent rendezvous protocol under discrete time in Equation (3), consider the position of the agent itself at the current time and the current position, predict the trend of the multi-agent movement, and take the prediction point as the virtual neighbor to participate in the next moment. Calculations, at this time the multi-agent aggregation protocol is

$$
\begin{aligned}
x_{i}(t+1) & =x_{i}(t)+\varepsilon\left(\sum_{j \in N_{i}} a_{i j}\left(x_{j}(t)-x_{i}(t)\right)+\psi_{i}\left(x_{i}(t-1)-x_{i}(t)\right)\right) \\
& =x_{i}(t)+\varepsilon\left(\sum_{j \in N_{i}} a_{i j}\left(x_{j}(t)-x_{i}(t)\right)-\varepsilon \psi_{i}\left(\sum_{j \in N_{i}} a_{i j}(t-1)\left(x_{j}(t-1)-x_{i}(t-1)\right)\right)\right)
\end{aligned}
$$

\section{MULTI-AGENT AGGREGATION BASED ON STATE BACKTRACKING}

The artificial potential field method is the most widely used method currently used to maintain topological connectivity. The method was first proposed by Kniab and was used early to avoid collisions between industrial robot arms [8]. In recent years, based on the potential function to maintain the topology connectivity, most of the results are concentrated on the continuous-time rendezvous protocol, and there are few researches on the aggregation problem under discrete time. Because the potential function controls the input to the infinite in the critical region, this range is not suitable for multi-agent systems that contain potential functions. Otherwise, the agents at the edge of the communication range may divergence due to excessive control input or cause the potential function to fail due to excessive adjustment factors. 

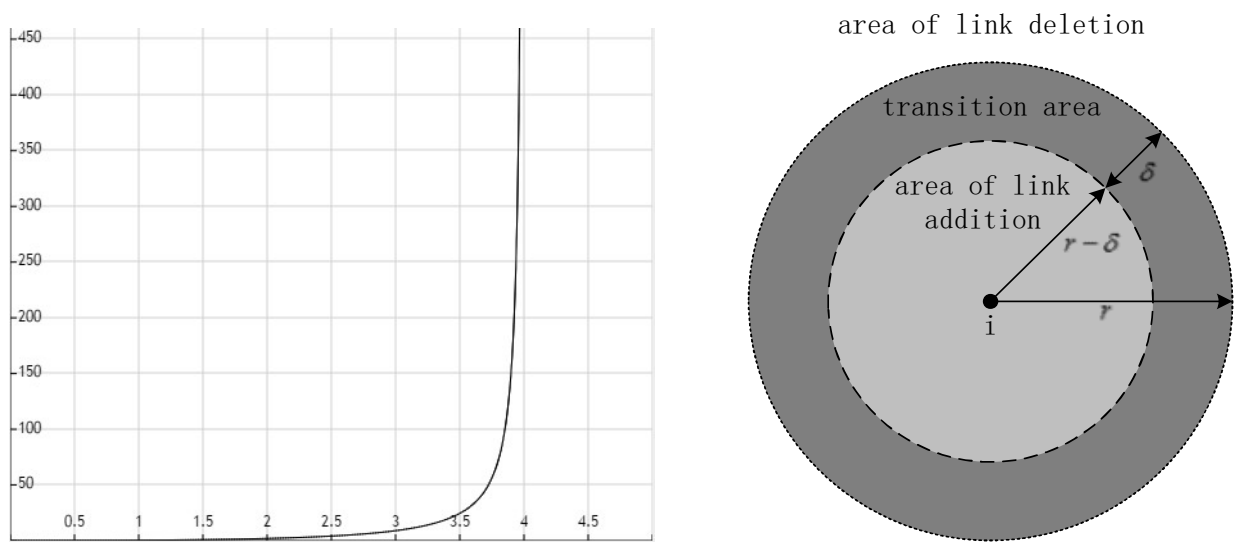

FIGURE 1. Potential function image

FIGURE 2. Setting of agent communication range area

As shown in the Fig.1, when $\left\|x_{i}-x_{j}\right\| \rightarrow r$ then $\varphi\left(\left\|x_{i}-x_{j}\right\|\right) \rightarrow \infty$, when the distance between agents increases, the control input also increases accordingly, which enhances the topological connectivity among agents.

By adding a potential control method to enhance topology connectivity, the challenge is mainly that when the distance between agents is in the critical area of the communication range during discontinuous time, the potential function increases, leading to system instability. References [8] and [10] have lagged behind the idea of establishing a connection, adding a transition zone in a multi-agent system to enhance the stability of the system.

As shown in the Fig.2, let $\delta$ be a positive real number and $\delta \in(0, r),\left\|x_{i}-x_{j}\right\|$ indicates the distance between the agent $i$ and the agent $j$. At the initial time edge set $E(0)=\left\{(i, j)\left\|x_{i}-x_{j}\right\|<r-\delta, i, j \in V\right\}$.If $(i, j) \notin E(t-1)$ and $0<\left\|x_{i}-x_{j}\right\|<r-\delta$, then $i$ enters the area to be connected to $i$ and joins $(i, j)$ to edge $\operatorname{set}(i, j) \notin E(t)$.If $\left\|x_{i}-x_{j}\right\|>r$, then $(i, j) \notin E(t)$.Therefore, the adjacency matrix $A=\left[a_{i j}\right]$ of the graph $G$ is expressed as:

$$
a_{i j}(t)=\left\{\begin{array}{l}
0, \text { if }\left(\left(a_{i j}(t-1)=0\right) \cap\left(\left\|x_{i}-x_{j}\right\| \geq r-\delta\right)\right) \cup\left(\left(a_{i j}(t-1)=1\right) \cap\left\|x_{i}-x_{j}\right\| \geq r\right) \\
1, \operatorname{if}\left(\left(a_{i j}(t-1)=1\right) \cap\left(\left\|x_{i}-x_{j}\right\|<r\right)\right) \cup\left(\left(a_{i j}(t-1)=0\right) \cap\left(\left\|x_{i}-x_{j}\right\| \leq r-\delta\right)\right)
\end{array}\right.
$$

The multi-agent rendezvous protocol design based on state backtracking is designed from two different perspectives respectively, and three state backtracking strategies are designed to speed up the rendezvous of multiagent and maintain topology connectivity. Inspired by [4] and [5], a potential function is added to the classical multiagent protocol:

$$
x_{i}(t+1)=x_{i}(t)+\varepsilon\left(H_{t}^{i}(x)-\varepsilon \psi_{i} H_{t-1}^{i}(x)\right)
$$

Where $H_{t}^{i}(x)=\sum_{j=1, j \neq i}^{n} a_{i j} \varphi\left(\left\|x_{i j}(t)\right\|\right) \operatorname{sign}\left(x_{j}(t)-x_{i}(t)\right)$ and $\varphi(x)$ is expressed as $\varphi\left(x_{i j}\right) \square \frac{x^{2}}{(r-x)}, x_{i j} \in[0, r)$.

Strategy 1:

The agent calculates the position of the current time $t$ and the time $t-1$, and obtains the estimated position $x_{i}(t+1)^{\text {pre }}$ of the time $t+1$, and takes the point as a virtual neighbor to participate in the calculation of the current time $t$.

Based on the study of Moreau [9], when the agent and the rest of the agents are fully connected and their positions are close to the same, the virtual neighbor based on the state backtracking setting may cause the convergence speed to decrease, so when the multi-agent approaches the rendezvous state, i.e., for any $j \in N_{i}$, when $\left\|x_{i j}\right\|<\partial$, no backtracking strategy is adopted. Therefore, the $\psi_{i}$ selection strategy in (12) is $\psi_{i}=0$, where $\left\|x_{i j}\right\| \leq \partial, j \in N_{i} \wedge i \neq j$, otherwise $\psi_{i}=1$

Under Strategy 1, through the prediction of the next moment in the historical state, it is possible to accelerate the convergence speed with a minimal adjustment factor. However, when the adjustment factor increases, the potential 
function may not guarantee topology connectivity. Therefore, when the neighbor agent is in a transitional area and there is a tendency to break away, use a switching strategy based on historical information.

Strategy 2:

It is necessary to avoid neighboring agents approaching critical areas as much as possible. Based on Strategy 1, add a state switch policy item $\psi_{i}$. The calculation flow is as follows:

TABLE 1. The switch policy item calculation flow

\begin{tabular}{|c|c|}
\hline Step 1) & Calculate the distance $\left\|x_{i j}(t)\right\|$ between agent $i$ and all neighbor agents. If for any $j \in N_{i},\left\|x_{i j}(t)\right\|<\partial$ then $\psi_{i}=0$ \\
\hline Step 2) & Calculate the set of neighbors common to agent $i$ at time $t$ and $t-1$, i.e. $S=\left\{j \mid j \in N_{i}(t) \wedge j \in N_{i}(t-1), i \neq j\right\}$ \\
\hline Step 3) & $\begin{array}{l}\text { Calculate the distance between agent } i \text { and the common neighbor set at } t \text { and } t-1 \text { respectively, i.e. }\left\|x_{i j}(t)\right\| \text { and }\left\|x_{i j}(t-1)\right\| \text {, } \\
\qquad j \in S\end{array}$ \\
\hline Step 4) & $\begin{array}{c}\text { Finding agent } i \text { satisfies }\left\|x_{i j}(t)\right\|-\left\|x_{i j}(t-1)\right\|>0 \text { and }\left\|x_{i j}(t)\right\|>r-\delta \text { in neighbor set } S_{j} \text { at time } t \text { and } t-1 \text {, i.e. a set of neighbor } \\
\text { agents that are in a transition area and are out of trend. }\end{array}$ \\
\hline Step 5) & Calculate the maximum distance $j_{\max }$ in the neighbor agent $\mathrm{j}$ that satisfies the condition of Step 4), i.e. $j=\arg \max \left(\left\|x_{i j}(t)\right\|\right)$. \\
\hline Step 6) & Calculate the position of the predicted point at time $\mathrm{t}+1$ (i.e. $x_{i}(t+1)^{p r e}$ ) by the position of agent $i$ at $t$ and $t-1$ \\
\hline Step 7) & $\begin{array}{c}\text { Assuming that } x_{i}(t+1)^{p r e} \text { is a virtual neighbor, that is, } \psi_{i}=1 \text {, the position } x_{i}(t+1)_{1} \text { of the agent } i \text { at the next moment is } \\
\text { calculated. }\end{array}$ \\
\hline Step 8) & Calculate the distance between $x_{i}(t+1)_{1}$ and $x_{i j_{\max }}(t)$, i.e. dist $=\left\|x_{i}(t+1)_{1}-x_{i j_{\max }}(t)\right\|$ \\
\hline Step 9) & $\begin{array}{l}\text { If } d i s t>r-\delta \text {, replace the agent } x_{i j}(t-1) \text { with } x_{i}(t+1)^{p r e} \text { as a virtual neighbor, that is, } \psi_{i}=-1 \text { is involved in the calculation of } \\
\text { the current time. }\end{array}$ \\
\hline
\end{tabular}

If the distance between the agent $i$ at the next moment and the agent $j$ at the current time exceeds the set threshold, they are regarded as having a tendency to detach. The agent's position at time $t-1$ is used as a virtual neighbor instead of the prediction point to participate in the calculation. The convergence rate of strategy 2 is relatively slow but can adapt to a larger adjustment factor.

\section{SIMULATION}

The simulation takes the agent radius $r=4$ and the length of the transition area as $\delta=0.5$. Take the example of 50 randomly distributed multi-agents in a circular region with a radius of 5.5. Assume that the initial distribution topology network is connected and the agents are randomly distributed in the plane. Two strategies to verify, determine the upper limit of the adjustment factor for multi-agent systems under different strategies, and analyze their performance.

\section{Model Parameters are Determined}

Because the selection range of adjustment factors in the traditional aggregation model does not apply to the multiagent rendezvous model with potential function. However, through experiments, it is found that the adjustment factor can ensure that the multi-agent system gathers at the same point within a certain range. Experiments were conducted on different adjustment factor values. The experimental results are shown in the following table.

TABLE 2. Adjustment Factor Experiment under Different Strategies

\begin{tabular}{|c|c|c|c|c|c|c|c|}
\hline $\begin{array}{l}\text { adjustment } \\
\text { factor }\end{array}$ & $\begin{array}{c}\text { Experiment } \\
\text { times }\end{array}$ & $\begin{array}{c}\text { Number of } \\
\text { Traditional } \\
\text { strategy } \\
\text { success }\end{array}$ & $\begin{array}{c}\text { Success rate } \\
\text { of Traditional } \\
\text { strategy }\end{array}$ & $\begin{array}{l}\text { Number of } \\
\text { strategy } 1 \\
\text { aggregation } \\
\text { success }\end{array}$ & $\begin{array}{l}\text { Success } \\
\text { rate of } \\
\text { strategy } 1\end{array}$ & $\begin{array}{l}\text { Number of } \\
\text { strategy } 2 \\
\text { aggregation } \\
\text { success }\end{array}$ & $\begin{array}{c}\text { Success } \\
\text { rate of } \\
\text { strategy } 2\end{array}$ \\
\hline 0.0002 & 50 & 50 & $100 \%$ & 50 & $100 \%$ & 50 & $100 \%$ \\
\hline 0.0003 & 50 & 50 & $100 \%$ & 50 & $100 \%$ & 50 & $100 \%$ \\
\hline 0.0004 & 50 & 49 & $98 \%$ & 50 & $100 \%$ & 50 & $100 \%$ \\
\hline 0.0005 & 50 & 49 & $98 \%$ & 49 & $98 \%$ & 50 & $100 \%$ \\
\hline 0.0006 & 50 & 48 & $96 \%$ & 48 & $96 \%$ & 49 & $98 \%$ \\
\hline 0.0007 & 50 & 47 & $94 \%$ & 47 & $94 \%$ & 49 & $98 \%$ \\
\hline 0.0008 & 50 & 46 & $92 \%$ & 46 & $92 \%$ & 49 & $98 \%$ \\
\hline
\end{tabular}


As shown in the above table, as the value of the adjustment factor increases, the success rate of rendezvous at the same point will decrease. Compared with the traditional model, Strategy 1 improves the robustness of the protocol and enhances topology connectivity by accelerating the convergence speed. Strategy 2 has added a new switching strategy based on Strategy 1, and the robustness has been further improved compared to the traditional model and Strategy 1.

Assume $\partial=0.35$, comparing Strategy 1 and Strategy 2, compared with the traditional model, the rate of increase of the final convergence of the multi-agent rendezvous model with potential function added.

TABLE 3. $\partial=0.35$ convergence rate increase rate

\begin{tabular}{ccc}
\hline Experiment times & speed increase rate of strategy 1 & speed increase rate of strategy 2 \\
\hline 20 & $24.05 \%$ & $21.76 \%$ \\
\hline
\end{tabular}

\section{Simulation Case}

The simulation takes 50 multi-agents as an example. Consider the initial multi-agent system network connectivity, the agent sensing radius $r=4$, the transition area $\delta=0.5$, the agent adjustment factor value $\varepsilon=0.0002$, the radius of the range where the multi-agent system switches to the retroactive state is $\partial=0.35$, the main consideration is to compare and analyze the rendezvous speeds of multi-agents randomly distributed in the plane under different strategies.

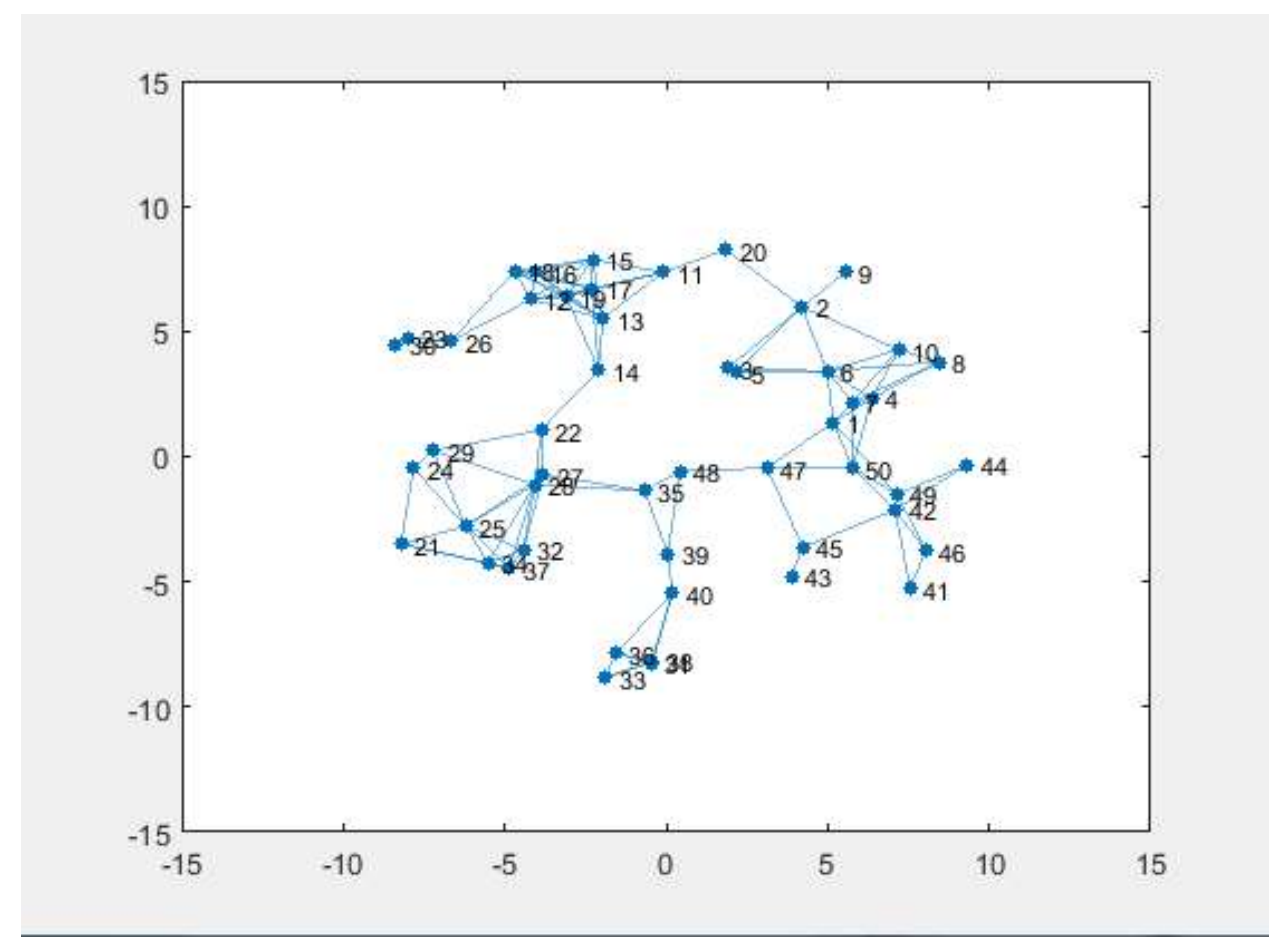

a) Initial network distribution 


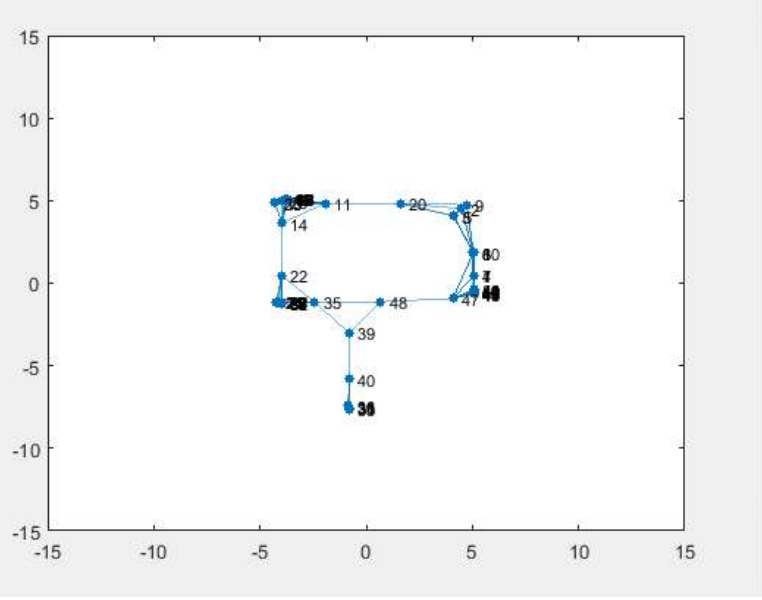

b) Agents are gathering

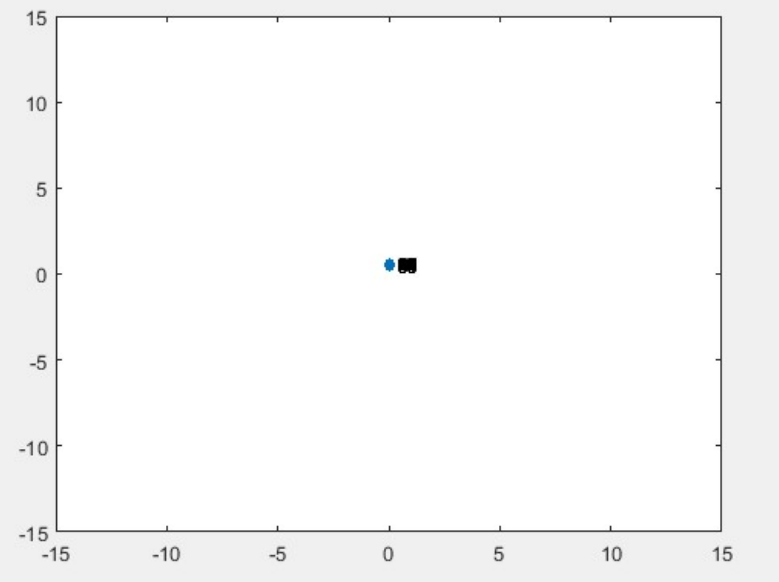

c) Final state

FIGURE 4. Agent evolution process

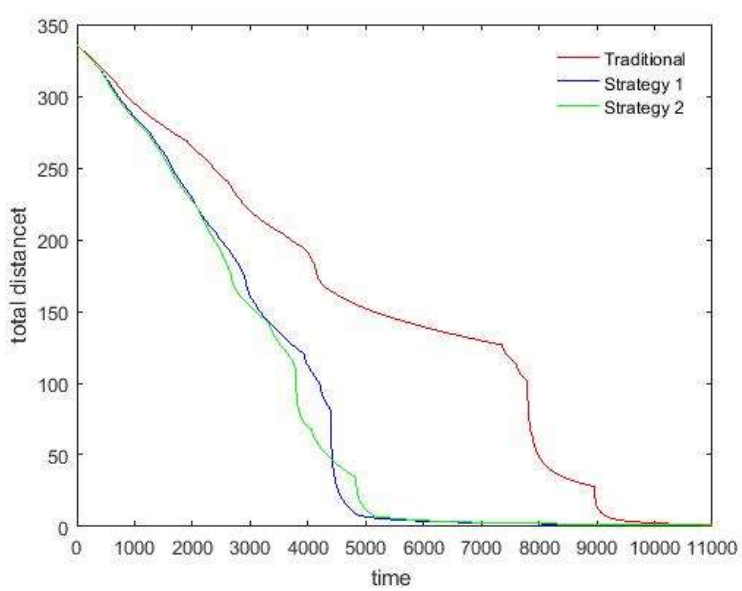

FIGURE 5. The curve of the total distance between agents 
As shown in the figure, multi-agent aggregation based on state backtracking has a faster convergence rate than the traditional multi-agent rendezvous problem, and it also has better results in topology connectivity.

\section{CONCLUSION}

This paper mainly studies the multi-agent rendezvous problem in discrete time based on state backtracking. In this problem, the state of the agent's history is used to evaluate the movement status of the agent's neighbors, so as to select the appropriate switching strategy. In addition, potential functions are also used to enhance the connectivity of the topology network. Because the traditional adjustment factor is not suitable for the current model, according to the experiment, the appropriate selection range of the adjustment factor is obtained and explained through simulation test.In future work, multi-agent rendezvous based on state backtracking under limited control inputs will be studied.

\section{REFERENCES}

1. Ando H,Suzuki I,Yamashita Formation and agreement problems for synchronous mobile robots with limited visibility [C]//IEEE International Symposium on Intelligent Control.IEEE,1995:453-460.

2. Lin J, Morse A S, Anderson B D O. The Multi-Agent Rendezvous Problem. Part 1: The Synchronous Case[J]. Siam Journal on Control \& Optimization, 2007, 46(6):1508 - 1513 Vol.2.

3. Dimarogonas D V, Johansson K H. Decentralized connectivity maintenance in mobile networks with bounded inputs[C]// IEEE International Conference on Robotics and Automation. IEEE,2008:1507-1512.

4. Hui Q.Finite-Time Rendezvous Algorithms for Mobile Autonomous Agents[J].IEEE Transactions on Automatic Control, 2011,56(1):207-211.

5. Su H,Wang X,Chen G. Rendezvous of multiple mobile agents with preserved network connectivity[J].Systems \& Control Letters, 2010,59(5):313-322.

6. Zavlanos M M, Pappas G J. Distributed Connectivity Control of Mobile Networks[J]. IEEE Transactions on Robotics, 2008, 24(6):1416-1428.

7. Suzuki I,Yamashita Distributed Anonymous Mobile Robots: Formation of Geometric Patterns[M].Society for Industrial and Applied Mathematics, 1999:28(4):279-280.

8. Khatib O. Real-Time Obstacle Avoidance for Manipulators and Mobile Robots[M]// Autonomous Robot Vehicles. Springer New York,1986:500-505.

9. Moreau L. Stability of multi-agent systems with time-dependent communication links[J]. IEEE Trans on Automatic Control, 2005, 50(2) 169-182. 